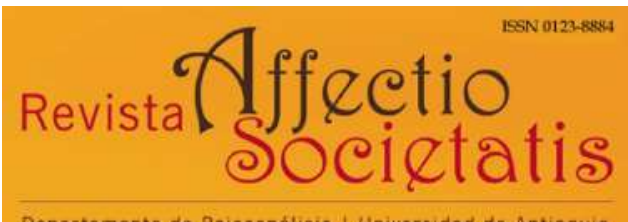

Departamento de Psicoanálisis I Universidad de Antioquia

Revista Affectio Societatis

Departamento de Psicoanálisis

Universidad de Antioquia

revistaaffectiosocietatis@udea.edu.co

ISSN (versión electrónica): 0123-8884

Colombia

2019

Maximiliano Cosentino

LA ANGUSTIA, MÁS ACÁ DEL CONOCIMIENTO

Revista Affectio Societatis, Vol. 16, N. ${ }^{\circ}$ 30, enero-junio de 2019

Art. \# 7 (pp. 132-143)

Departamento de Psicoanálisis, Universidad de Antioquia

Medellín, Colombia 


\title{
LA ANGUSTIA, MÁS ACÁ DEL CONOCIMIENTO
}

\author{
Maximiliano Cosentino ${ }^{1}$ \\ Universidad de Buenos Aires, Argentina \\ maximiliano.cosentino@gmail.com \\ ORCID: 0000-0002-6098-211X
}

DOI: 10.17533/udea.affs.v16n30a07

\section{Resumen}

En el presente artículo nos proponemos abordar un aspecto escasamente trabajado en la bibliografía lacaniana, a saber, la recepción de la Crítica de la razón pura -especialmente, "La estética trascendental" - en el seminario La angustia de Jacques Lacan. Efectivamente, los comentadores de la obra de Lacan se han centrado casi de manera exclusiva en la recepción de la filosofía práctica de Kant que ha realizado

el psicoanalista francés, dejando de lado la importancia de la Crítica de la razón pura para delimitar al objeto a. En este punto, será necesario evocar la distinción fundamental entre objetividad y objetalidad para distinguir, así, el deseo a la base de las condiciones de posibilidad del conocimiento al deseo.

Palabras claves: objetividad, objetalidad, epistemología, Kant, objeto a.

\section{ANXIETY, ON THIS SIDE OF KNOWLEDGE}

\section{Abstract}

This paper aims at addressing one aspect barely studied of the Lacanian bibliography, i.e., the reception of the Critique of Pure Reason -especially, the "Transcendental Aesthetic" - in Jacques Lacan's seminary on Anxiety. Indeed, commentators of Lacan's work have almost exclusively fo- cused on his reception of Kant's practical philosophy, putting aside the importance of the Critique of Pure Reason to delimit the object a. In this point, it will be necessary to recall the fundamental difference between objectivity and objectality in order to distinguish the desire on the base

1 Licenciado en Psicología (UBA). Becario doctoral UBACyT. Docente e investigador (UBA/UAI). Director de la revista Litura (UAI). 
of the conditions of possibility from Keywords: objectivity, objectality, knowledge to desire.

epistemology, Kant, object a.

\section{L'ANGOISSE, EN DEÇÀ DE LA CONNAISSANCE.}

\section{Résumé :}

Cet article aborde un aspect peu étu- l'importance de la Critique de la raidié de l'œuvre lacanienne, à savoir, son pure pour délimiter l'objet a. Sur la réception de la Critique de la rai- ce point, il est nécessaire d'évoquer la son pure - notamment "L'esthétique transcendantale «- dans le séminaire L'angoisse de Jacques Lacan. En effet, les commentateurs de l'œuvre de Lacan se sont focalisés presque exclusivement sur la réception de la philosophie pratique de Kant de la part du psychanalyste français, en oubliant distinction fondamentale entre objectivité et objectalité, pour différencier ainsi le désir à la base des conditions de possibilité de la connaissance au désir.

Mots-clés : objectivité, objectalité, épistémologie, Kant, objet a.

Recibido:10/04/2018 • Aprobado:10/06/2018 
"Lo que evoco aquí para ustedes no es metafísica. Es más bien un lavado de cerebro" Lacan, Seminario 10: La angustia.

A lo largo de sus escritos y seminarios, Lacan estableció un diálogo constante con la filosofía trascendental de Kant. Aún más, se podría sostener, sin riesgo de exagerar, que Kant - junto con Aristóteles, Platón, Descartes y Hegel - es uno de los filósofos más nombrados por el psicoanalista francés. De hecho, Kant es el único que ostenta el privilegio de intitular uno de los textos que conforman los Escritos, como es sabido, el célebre "Kant con Sade" (1963). Ahora bien, al examinar las menciones a la obra kantiana - basta en este punto indicar los casos paradigmáticos, como el escrito anteriormente señalado o el seminario del año lectivo 1959-1960 sobre La ética del psicoanálisispodemos constatar que la lectura de Lacan se centra casi exclusivamente en su vertiente práctica ${ }^{2}$. En esta dirección también podemos situar los desarrollos en el seminario del año 1962-1963 sobre La angustia en el que Lacan, a través del objeto voz, intenta dar cuenta del imperativo categórico ${ }^{3}$ y de su articulación con el superyó. Sin embargo, además de las referencias a la filosofía práctica kantiana, a lo largo del seminario encontramos, de forma insistente, menciones más o menos directas a la Crítica de la razón pura y especialmente a la estética trascendental. La introducción de estas referencias a la teoría del conocimiento de Kant se explica dado que gran parte de las reflexiones del Seminario La angustia están dirigidas a situar la especificidad del objeto $a$. De este modo, la Crítica de la razón pura funcionará a lo largo del seminario como telón de fondo sobre el cual confrontar la dimensión del objeto en psicoanálisis. Para dar cuenta de estos movimien-

2 Ésta vía de lectura centrada en la recepción de la filosofía práctica kantiana en la obra de Lacan se reproduce en sus comentaristas. Basta, como ejemplo, citar el libro de Zupančič (2012) Ethics of the Real.

3 Así lo indica: “(...) cierto imperativo llamado categórico, en el que encontramos de nuevo el carácter de certeza fundamental ya advertido por la filosofía tradicional y articulado por Kant bajo la forma de la conciencia moral. Abordarlo desde la perspectiva del $a$ nos permite situarlo en su lugar" (2006/1962-1963, p. 249). 
tos, en primer lugar, vamos a caracterizar los puntos fundamentales del proyecto kantiano en la Crítica de la razón pura poniendo énfasis en el giro copernicano producido en la relación entre el sujeto y el objeto; en segundo lugar, confrontaremos la perspectiva kantiana con el esquema de la división subjetiva presentado por Lacan durante el Seminario La angustia; en tercer lugar, siguiendo la distinción lacaniana entre objetividad y objetalidad, mostraremos la función del fantasma para el conocimiento; finalmente, propondremos una lectura ética del objeto $a$ para evitar interpretaciones de corte epistemológico.

\section{Las condiciones de posibilidad de la objetividad}

Como se sabe, en el segundo prólogo a la Crítica de la razón pura, Kant propone realizar una "revolución copernicana" en la metafísica (B XVI). En otras palabras, producir en metafísica el cambio de perspectiva que Copérnico estableció para pensar los fenómenos astronómicos, es decir, el abandono de la perspectiva natural que aparenta que el Sol gira alrededor de la Tierra por la perspectiva de que la Tierra (y demás planetas) gira alrededor del Sol. En este sentido, el gesto que va a rescatar Kant de la revolución copernicana no es la refutación de la cosmología aristotélico-ptolemaica, sino el cambio de perspectiva que supuso ${ }^{4}$. Esta modificación de perspectiva es la que Kant persigue para la metafísica, en otros términos, "en la metafísica se puede hacer un ensayo semejante, en lo que concierne a la intuición de los objetos" (B XVII). Es conocido el cambio de perspectiva que introduce Kant en la Crítica de la razón pura para la metafísica: se abandona la perspectiva que considera que el conocimiento debe adecuarse a los objetos por la perspectiva que los objetos deben ade-

4 Como ya bien ha señalado Koyré en Del mundo cerrado al universo infinito (2002/1957, pp. 31-40), la revolución de Copérnico no fue un corte absoluto con la cosmología aristotélico-ptolemaica. De hecho, compartía varios de sus supuestos, los más notorios: el carácter finito del universo y su forma esférica. De este modo, la característica esencial de la revolución copernicana hay que ubicarla en el cambio de perspectiva que, por supuesto, tuvo un efecto abrumador y radical en la ciencia y la filosofía. 
cuarse al conocimiento. De este modo, Kant va a distinguir dos factores esenciales en el acto de conocer, dado que "pensamientos sin contenidos son vacíos, intuiciones sin conceptos son ciegas" (A51/ B75): por un lado, la estructura de la razón; por el otro, las impresiones o sensaciones.

Dicho de manera más precisa, desde la perspectiva kantiana, la razón está compuesta por las formas puras de la sensibilidad - el espacio y el tiempo - que son tematizadas en la "Estética trascendental" y por las categorías o formas puras del entendimiento que son desarrolladas en la "Lógica trascendental". En este sentido, las formas puras de la sensibilidad - el espacio y el tiempo- y las formas puras del entendimiento - las categorías - no son propiedades de los objetos, sino que conforman la estructura a priori del sujeto que permite la posibilidad que se elaboren los objetos. Sin embargo, para que pueda darse un objeto son necesarias las impresiones o sensaciones. De esta manera, si se intentase conocer mediante las formas puras de la razón, sean de la sensibilidad o del entendimiento, solamente tendríamos formas vacías sin contenido. Es necesario que las formas a priori del sujeto se apliquen a las impresiones y sensaciones que son dadas en la experiencia. De manera complementaria, las impresiones y sensaciones sin formas puras, es decir, sin la estructura a priori del sujeto, son un caos sin orden.

La primera consecuencia que se puede extraer del planteo kantiano es que solamente puede haber conocimiento dentro de los límites de una experiencia dada a un sujeto; en otras palabras, solo puede haber conocimiento de los fenómenos. En este sentido preciso, el conocimiento metafísico queda imposibilitado - de esto se trata la crítica como límite de la razón pura - , ya que los conceptos, siguiendo el paradigma kantiano, de Dios, alma y mundo no nos son dados en la experiencia. De este modo, Kant postulará en la "Dialéctica trascendental" que Dios, alma y mundo son solo ideas regulativas de la razón; en otras palabras, las ideas solamente pueden ser pensadas, pero nunca conocidas. Una segunda consecuencia del planteo kantiano, ligada a la primera, aunque más relevante para los fines de nuestro trabajo, es que el objeto se encuentra siempre en relación con el sujeto; tal como lo hemos expresado anteriormente, en el sujeto se encuen- 
tran las condiciones de posibilidad de la objetividad o, dicho de otra forma, solo podemos conocer los objetos en cuanto fenómeno (el objeto "para-mí" $i^{\prime \prime}$ y nunca en tanto noúmeno o como cosa en sí (Das Ding an sich). Por lo tanto, al depender el objeto -y su objetividad - de una teoría del sujeto - en cuanto condición de posibilidad de toda experiencia de objeto posible - , se impide que pueda existir una ontología autónoma, más precisamente, una ontología de lo absoluto, es decir, que no sea relativa al sujeto, o como responde Badiou a una pregunta de un estudiante en una lección en The European Graduate School: "Si sostienes que el objeto, y finalmente la objetividad, está necesariamente en relación con un sujeto entonces estás bajo la visión de que la objetividad depende de la subjetividad. Y esa es la visión de Kant" ${ }^{\prime \prime}$ (2012, pp. 103-104).

\section{Un objeto que escapa a la estética trascendental}

En la tercera clase del seminario sobre la angustia, luego de presentar el primer esquema de la división subjetiva, Lacan se apresura a precisar que el objeto $a$ es un objeto que "escapa a las leyes de la estética trascendental" (2006/1962-1963, p. 50). En este punto, es necesario recordar, para situar la diferencia sustancial del planteo lacaniano con el kantiano, la operación de la división del sujeto. En un primer nivel, ${ }^{7}$ caracterizado como "mítico" (2006/1962-1963, p. 175), el sujeto (S) debe constituirse en el lugar del Otro (A) -entendido como "tesoro de los significantes" (p. 175) - . El sujeto (S), al intentar situarse en el Otro (A) mediante la forma de una pregunta sobre su deseo -el caso paradigmático es el Che vuoi? - , transforma al Otro completo -insisti-

5 Tomamos prestada la expresión “objeto para-mí" de Después de la finitud (2006), justamente allí Meillassoux critica fuertemente a la filosofía kantiana.

6 Traducción del autor de este trabajo. Versión original en inglés: “If you claim that the object, and finally objectivity, is by necessity in a relationship to a subject then you are within the vision that claims that objectivity depends on subjectivity. And so it is the Kantian vision".

7 Preferimos conservar la expresión de Lacan, niveau mythique (nivel mítico), que consideramos evita las interpretaciones de corte genético con la temporalidad lineal que le es propia. 
mos en su carácter mítico - en Otro barrado (A). El resultado de esta operación del sujeto (S) sobre el Otro (A) se expresa en el segundo nivel, donde la diferencia entre las dos formas del Otro, es decir entre A y $\mathrm{A}$, se escribe, mediante la notación lacaniana, como $a$. De esta manera, el $a$ es, ante todo, un resto irreductible producto de la primera interrogación del sujeto (S) al Otro (A). Sin embargo, es recién en un tercer nivel donde la división del sujeto se consuma, dado que el $a$ tendrá la función de representar al sujeto (S) en su aspecto real, de esta forma, lo convierte en sujeto dividido (\$). Lacan esquematiza la operación de la división subjetiva del siguiente modo

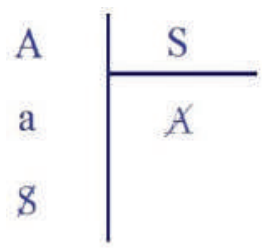

Figura 1. Primer esquema de la división.

Fuente: Tomada de: Lacan, J. El seminario, Libro 10: La angustia (p. 36).

De este esquema podemos extraer algunas conclusiones importantes para entender la necesidad de Lacan de evocar la estética trascendental como contraste para su teorización sobre el $a$. En primer lugar, salta a la vista que tanto el $a$ como el $\$$ quedan ubicados del lado del Otro (A) -lado que Lacan había denominado, en la segunda clase del seminario, el "lado objetivo de la barra" (2006/1962-1963, p. 36) - y, en consecuencia, también lo está el fantasma \$<>a. En segundo lugar, el objeto $a$ se constituye como un resto o residuo de la operación de la división del sujeto. En este punto, el objeto $a$ no se confunde con ningún objeto "contable" o de "intercambio" (p.103), en otras palabras, el objeto $a$ no es un objeto intramundano. Aunque aquí conviene ser preciso: tampoco se trata de un objeto por fuera del mundo, que resulta inaccesible al sujeto pero que es necesario suponer. El objeto $a$ no se debe confundir con la cosa en sí o el noúmeno kantiano, dado que no es el

8 En sentido estricto, se trata del tercer esquema de la división subjetiva dado que Lacan ya había presentado otros dos con anterioridad en el seminario de $\mathrm{La}$ angustia. Si bien presentan diferencias, por los límites de este trabajo, pondremos nuestra atención en éste que es más cercano a nuestro propósito argumentativo. 
objeto sin el sujeto, es decir, la cosa; sino que el objeto $a$ entraña una relación específica con el sujeto: por un lado, es el resto de su producción; por el otro, es su representante ante el Otro. En este sentido, estamos de acuerdo con lo indicado por Harari en su introducción a la lectura del seminario La angustia, en cuanto que el esquema de la división del sujeto no expresa la relación de un sujeto ante un objeto. Aunque no coincidimos con el aspecto positivo de su interpretación, dado que Harari considera que la operación de la división del sujeto supone una fractura de la "epistemología clásica (...) el positivismo" (1993, p. 53) que implica una ruptura con la ciencia. Sin embargo, este último punto implica confundir una discusión epistemológica -en el sentido de qué se puede conocer - con una discusión sobre el estatuto científico del psicoanálisis -cuál es su lugar dentro de la ciencia - ; pero, aún más, es perder de vista que el objeto $a$ en Lacan no está relacionado con ningún tipo de teoría del conocimiento (y no sólo con el positivismo) porque, como hemos indicado con anterioridad, se trata de un objeto particular producido en la constitución subjetiva que no tiene entidad -se trata, más bien, de una falta irreductible - y no, en términos heideggerianos, de un objeto a la mano (Zuhandenheit). Por nuestra parte, no podemos dejar de señalar, junto a Lacan, que el psicoanálisis se sitúa "en un más acá anterior al momento del conocimiento" (2006/1962-1963, p. 67), en otras palabras, el psicoanálisis no es una epistemología o teoría del conocimiento. Este punto es enfatizado por Lacan en la clase del 9 de enero de 1963:

\section{(...) designar esta $a$ minúscula con el término objeto es hacer un uso metafórico de dicha palabra, porque está tomada de la relación sujeto-objeto (...) de lo que nosotros tenemos que hablar haciendo uso del término $a$ es precisamente de un objeto externo a toda defi- nición posible de objetividad (xxxx2006/1962-1963, p. 98).}

Nuevamente, en el transcurso de esta clase, Lacan va a aludir a Kant para tomar distancia de su estética trascendental. Creemos que esta insistencia se debe a dos motivos. El primero, más general, a clarificar, como venimos señalando, que no está construyendo una teoría del conocimiento. El segundo, más particular, a mostrar que si bien está postulando una relación entre el sujeto y el objeto, no es al modo kantiano -es decir, en tanto que en el sujeto están las condiciones de 
posibilidad de la objetividad-, sino que la única relación posible entre el sujeto (\$) y el objeto $a$ es fantasmática y no implica conocimiento, sino el marco para el deseo. La conjunción de estos dos puntos redunda en la distinción lacaniana entre objetividad y objetalidad.

\section{¿Objetividad u objetalidad?}

En el tramo final del Seminario La angustia, Lacan profundiza sus diferencias con Kant y precisa para el objeto $a$ "el término de objetalidad como opuesto al de objetividad" (xxxx2006/1962-1963, p. 232). De esta manera, Lacan insiste en no identificar al objeto $a$ como el correlato objetivo del sujeto -tal como se plantea en la Crítica de la razón pura-, sino que propone entender al $a$ bajo la rúbrica de la objetalidad, dicho de otra forma, como "el correlato de un pathos de corte" (xxxx/p.232). En un giro sorpresivo, Lacan introduce el problema de la causalidad en Kant, a quien considera "repleto de causalidad" (p.233). En efecto, Kant, en la Crítica de la razón pura establece como una de las formas puras del entendimiento -es decir, como una categoría- la relación de causalidad entre fenómenos ${ }^{9}$; por lo tanto, la relación de causalidad forma parte de las condiciones de posibilidad de la objetividad del sujeto. Ahora bien, ¿cómo se relaciona el problema de la causalidad con el objeto $a$ ?

En este punto debemos recordar que el psicoanálisis se ubica en un momento lógico previo al del conocimiento, por lo que la hipótesis fuerte de Lacan será que a la base de cualquier tentativa de construir una teoría del conocimiento -Kant es el caso paradigmático en el Seminario La angustia - se encuentra operando el $a$. Más precisamente, la objetalidad como pathos del corte -es decir, como afecto que acompaña a la división del sujeto- opera como causa del deseo de conocimiento. Desde la perspectiva lacaniana, en la causalidad no se trata de otra cosa que de ese "pedazo carnal arrancado de nosotros mismos" (2006/19621963, p. 233) que cumple la función de ser "la tripa causal" (p. 234)

9 En este punto debemos aclarar que el problema de la causalidad es abordado por Kant en la Crítica de la razón pura dentro del contexto de las antinomias de la razón pura en el apartado de la "Dialéctica trascendental". 
para el deseo de conocimiento. De esta manera, la apuesta de Lacan es radical, ya que la angustia es la certeza que se encuentra a la base del deseo de conocer. Este punto, por otra parte, es coherente con lo señalado en el esquema de la división subjetiva, a saber, que la angustia tiene una posición media entre el goce y el deseo; por lo tanto, solo se puede acceder al deseo habiendo atravesado la angustia. Una consecuencia interesante que se deduce de este planteo es que, en última instancia, la certeza de la angustia -vale recordar que Lacan la vinculó con lo que no engaña- es la causa de la búsqueda de certezas metafísicas o, de otro modo, del deseo de conocimiento. Por este motivo, Lacan sostiene que el Dios de San Anselmo, el de Descartes o la causa primera de Aristóteles, en fin, los conceptos que funcionan como causa (arché) y principio (aitias) en los diversos sistemas metafísicos occidentales, son producto del $a$ obtenido por la división subjetiva. En este sentido, el concepto que opera como fundamento es un desplazamiento de su verdadero fundamento, es decir, la angustia.

No es exagerado sostener que Lacan se ubica en la línea, abierta por Heidegger, de pensadores críticos de la metafísica de la presencia; o al menos no más exagerado que Lacan al afirmar que estaba realizando "el cuestionamiento más radical que jamás se haya articulado en nuestra filosofía occidental de la función del conocimiento" (2006/1962-1963, p. 236). En esta dirección se vuelve clara la función del fantasma; efectivamente, Lacan afirma que en el fantasma ya hay conocimiento porque con el nivel del fantasma nos encontramos en el marco del deseo o, aún más, el fantasma es una pantalla ante la angustia -esto es lo que expresa el matema $\$<>a-$, en otras palabras, el fantasma es un momento posterior al de la angustia. Por lo que se podría afirmar, siguiendo la pista abierta por Lacan, que todo deseo de conocimiento es, en última instancia, fantasmástico puesto que se trata de una reacción defensiva ante la angustia.

\section{Hacia una ética trascendental}

Llegando al final del seminario sobre La angustia, Lacan sostiene que el $a$ como causa del deseo es una extracción del funcionamiento de la causa del dominio de la estética trascendental al de la "ética tras- 
cendental" (p. 304). Más allá del carácter irónico de esta afirmación -el término "trascendental" refiere en Kant exclusivamente al conocimiento $^{10}-$, Lacan estaría afirmando que la posición del sujeto ante el $a$-el fantasma- es una respuesta ante la inconsistencia del Otro (A). En esta dirección, en el seminario del año lectivo 1958-59, El deseo y su interpretación, Lacan sitúa al fantasma como el lugar donde "el sujeto encuentra su soporte en el momento en que se desvanece ante la carencia del significante que responda por su lugar de sujeto en el nivel del Otro" (2014/1958-1959, p. 418). En este preciso sentido, el fantasma es una pantalla, una respuesta o una forma de estructurar la realidad del deseo que sostiene al sujeto en fading ante el encuentro con el aspecto real del Otro, es decir, su incompletud. De este modo, el fantasma es una protección ante lo real, pero, simultáneamente, lo real es soporte del fantasma. En otras palabras, el fantasma es una forma de velar la verdad paradójica del sujeto, es decir, su falta en ser (manque à être); pero, al mismo tiempo, su falta en ser enfrentada con la falta del Otro (escrito como $a$ ) encuentran su coagulación en el fantasma. De este modo, el estatuto del objeto $a$, en cuanto solicita una respuesta, es ético más que epistemológico.

\section{Referencias}

Badiou, A. (2012). The Subject of Change. New York, E.U.: Athropos Press.

Harari, R. (1993). El seminario "La angustia" de Lacan: una introducción. Buenos Aires, Argentina: Amorrortu.

Kant, I. (2009/1781). Crítica de la razón pura. Buenos Aires, Argentina: Colihue. Koyré, A. (2002/1957). Del mundo cerrado al universo infinito. Buenos Aires, Argentina: Siglo Veintiuno Editores.

Lacan, J. (2014/1958-1959). El seminario, Libro 6: El deseo y su interpretación. Buenos Aires, Argentina: Paidós.

Lacan, J. (2006/1962-1963). El seminario, Libro 10: La angustia. Buenos Aires, Argentina: Paidós.

10 Así lo expresa Kant en la Crítica de la razón pura: "Llamo trascendental todo conocimiento que se ocupa, en general, no tanto de objetos, como de nuestra manera de conocer los objetos, en la medida que ella ha de ser posible a priori" (A12/B25). 
Lacan, J. (2001/1963). “Kant con Sade”. En Escritos 2. Buenos Aires, Argentina: Siglo Veintiuno Editores.

Meillassoux, Q. (2016/2006). Después de la finitud. Ensayos sobre la necesidad de la contingencia. Buenos Aires, Argentina: Caja Negra.

Zupančič, A. (2012). Ethics of the Real. New York, E.U.: Verso. 